\title{
Numerical Simulation of Three-Dimensional Vertically Aligned Quantum Dot Array
}

\author{
Weichung Wang ${ }^{1}$ and Tsung-Min Hwang ${ }^{2}$ \\ 1 Department of Applied Mathematics, National University of Kaohsiung, \\ Kaohsiung 811, Taiwan \\ wwang@nuk.edu.tw \\ 2 Department of Mathematics, National Taiwan Normal University, \\ Taipei 116, Taiwan \\ min@math.ntnu.edu.tw
}

\begin{abstract}
We study the electronic properties of quantum dot arrays formed by 2 to 12 vertically aligned quantum dots numerically. Numerical schemes in grid points choosing, finite differences, matrix reduction, and large-scale eigenvalue problem solver are discussed. The schemes allow us to compute all the desired energy states and the wave functions efficiently. Numerical experiment results are presented.
\end{abstract}

Keywords: Semiconductor quantum dot array, the Schrödinger equation, energy levels, wave function, numerical simulation.

\section{Vertically Aligned Quantum Dot Array Model}

Recent advances in fabrication and varied applications of semiconductor quantum dot array (QDA) have attracted intensive studies in theoretical, experimental, and numerical. The energy state spectrum and the corresponding wave functions of a QDA system is of basic physical interest and is crucial for designing applications like photoelectric devices.

A main challenge for simulating a three-dimensional QDA is to solve very large scale eigenvalue problems for only several interior eigenvalues that are of interest. Aiming at the QDA that disk-shaped co-axial InAs QDs are vertically aligned and embedded in a cylindrical GaAs matrix (see left part of Figure 10), we develop efficient numerical schemes overcome the difficulties.

The QDA is modelled by the Schrödinger equation, in the cylindrical coordinate, as

$$
\frac{-\hbar^{2}}{2 m_{\ell}(\lambda)}\left[\frac{\partial^{2} F}{\partial r^{2}}+\frac{1}{r} \frac{\partial F}{\partial r}+\frac{1}{r^{2}} \frac{\partial^{2} F}{\partial \theta^{2}}+\frac{\partial^{2} F}{\partial z^{2}}\right]+c_{\ell} F=\lambda F,
$$

where $\hbar$ is the reduced Plank constant, $\lambda$ is the total electron energy, $F=$ $F(r, \theta, z)$ is the wave function, $m_{\ell}(\lambda)$ and $c_{\ell}$ are the electron effective mass and confinement potential in the $\ell$ th region. The index $\ell$ is used to distinguish the 

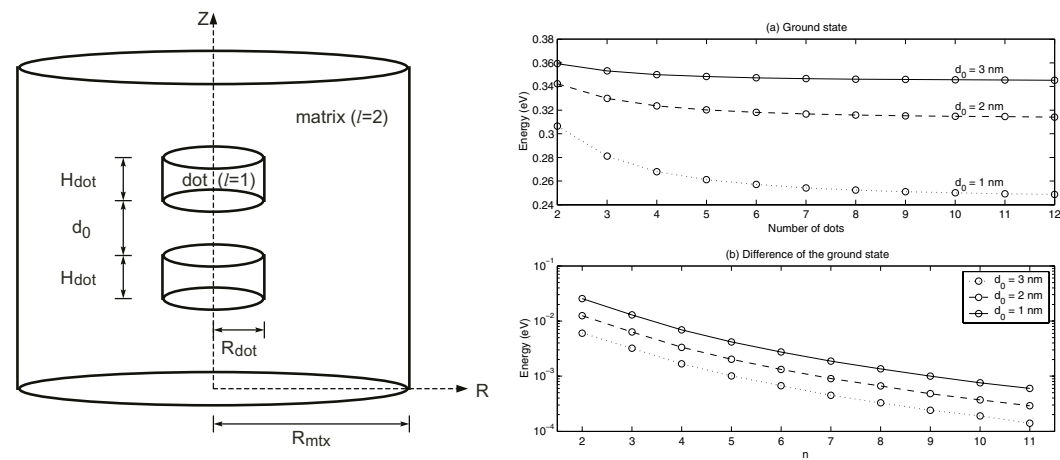

Fig. 1. Left part: Structure schema of a cylindrical vertically aligned quantum dot array and the heterostructure matrix. Right part: Ground state energies for various spacer layer distances $d_{0}$ and number of quantum dots

region of the QDs (for $\ell=1$ ) from that of the matrix (for $\ell=2$ ). The nonparabolic effective mass approximation [1] is given as

$$
\frac{1}{m_{\ell}(\lambda)}=\frac{P_{\ell}^{2}}{\hbar^{2}}\left(\frac{2}{\lambda+g_{\ell}-c_{\ell}}+\frac{1}{\lambda+g_{\ell}-c_{\ell}+\delta_{\ell}}\right),
$$

where $P_{\ell}, g_{\ell}$, and $\delta_{\ell}$ are the momentum, main energy gap, and spin-orbit splitting in the $\ell$ th region, respectively. For Eq. (1), the Ben Daniel-Duke interface conditions [2] are imposed on the interface of the two different materials as

$$
\frac{\hbar^{2}}{2 m_{1}(\lambda)} \frac{\partial F\left(r_{I}, \theta_{I}, z_{I}\right)}{\partial n_{-}}=\frac{\hbar^{2}}{2 m_{2}(\lambda)} \frac{\partial F\left(r_{I}, \theta_{I}, z_{I}\right)}{\partial n_{+}}
$$

where $\left(r_{I}, \theta_{I}, z_{I}\right)$ denotes the position on the interface and the $n_{+}$and $n_{-}$denote the corresponding outward normal derivatives on the interface. Finally, Dirichlet boundary conditions are prescribed on the boundary (top, bottom, and wall) of the matrix.

\section{$2 \quad$ Numerical Schemes}

This section discusses the numerical schemes used for solving the threedimensional QDA Schrödinger equation (11) to compute the electron energy levels and the associated wave function in the system.

We first discretize the domain by choosing mesh points. Regular uniform mesh points are chosen in the azimuthal angle $\theta$ coordinate. Non-uniform mesh points are used in the radial coordinate $r$ and the natural axial coordinate $z$ with the following two special treatments. First, in the heterojunction area, fine meshes are used to capture the rapid change of the wave functions. Secondly, a half of the mesh length is shifted in the radial coordinate to avoid incorporating the pole condition [3]. 
Based on the grid points, Eq. (1) is discretized by the 3D centered seven-point finite difference method

$$
\begin{aligned}
& \frac{-\hbar^{2}}{2 m_{\ell}(\lambda)}\left(\frac{F_{i+1, j, k}-2 F_{i, j, k}+F_{i-1, j, k}}{(\Delta r)^{2}}+\frac{1}{r_{i}} \frac{F_{i+1, j, k}-F_{i-1, j, k}}{2 \Delta r}\right. \\
& \left.+\frac{1}{r_{i}^{2}} \frac{F_{i, j+1, k}-2 F_{i, j, k}+F_{i, j-1, k}}{(\Delta \theta)^{2}}+\frac{F_{i, j, k+1}-2 F_{i, j, k}+F_{i, j, k-1}}{(\Delta z)^{2}}\right)+c_{\ell} F_{i, j, k}=\lambda F_{i, j, k},
\end{aligned}
$$

where $F_{i, j, k}$ is the approximated value of wave function $F$ at the grid point $\left(r_{i}, \theta_{j}, z_{k}\right)$ for $\ell=1,2, i=1, \ldots, \rho, j=1, \ldots, \mu$, and $k=1, \ldots, \zeta$. In the heterojunctions, two-point finite differences are applied on the interface conditions of the QDs. The numerical boundary values for the matrix in the $z$ - and $r$-direction are zeros according to the Dirichlet boundary conditions.

Assembling the finite difference discretizations results in a $\rho \mu \zeta$-by- $\rho \mu \zeta 3 \mathrm{D}$ eigenvalue problem. By reordering the unknown vector and using the fast Fourier transformation to tridiagonalize matrices $T_{k}(\lambda)$ (for $k=1, \ldots, \zeta$ ), we obtain $\mu$ independent $\rho \zeta$-by- $\rho \zeta$ eigenvalue problems with the form

$$
\widetilde{T}_{j}(\lambda) \widetilde{F}_{j}=\widetilde{D}_{j}(\lambda) \widetilde{F}_{j},
$$

for $j=1, \ldots, \mu$, where $\widetilde{T}_{j}(\lambda)$ and $\widetilde{D}_{j}(\lambda)$ are $\rho \zeta$-by- $\rho \zeta$ matrices. Each of the eigenvalue problems in the form of (4) is called a $2 \mathrm{D}$ eigenvalue problem, since the grid points of the unknowns in $\widetilde{F}_{j}$ have the same $\theta$ value. By multiplying the common denominator of (4), we can then form the cubic $\lambda$-matrix polynomial

$$
\mathbf{A}(\lambda) \mathbf{F}=\left(\lambda^{3} A_{3}+\lambda^{2} A_{2}+\lambda A_{1}+A_{0}\right) \mathbf{F}=0,
$$

where $A_{0}, A_{1}, A_{2}$, and $A_{3}$ are independent to $\lambda$. The cubic eigenvalue problem can then be solved efficiently by (i) the cubic Jacobi-Davidson method to compute the smallest positive eigenvalue representing the ground state energy, and (ii) the explicit deflation scheme to estimate the successive smallest positive eigenvalues (i.e. the excited energy states). See [4,5] for detail.

\section{$3 \quad$ Results and Discussions}

In our numerical experiments, we assume that $H_{d o t}$ and $R_{d o t}$ of the QDs are 3 and $7.5 \mathrm{~nm}$, respectively. For the matrix, $R_{m t x}=37.5 \mathrm{~nm}$ and $6 \mathrm{~nm}$ matrix layer are assumed above the top and below the bottom of the QDA. The material parameters used in the experiments are $c_{1}=0.0000, g_{1}=0.4200, \delta_{1}=0.4800$, $P_{1}=0.7730, c_{2}=0.7700, g_{2}=1.5200, \delta_{2}=0.3400$, and $P_{2}=0.8071$. Numerical simulation findings are summarized as follows.

The ground state energy of the QDA are affected by the number of QDs and the spacer layer distances $d_{0}$. Right top of Figure 1 (a) shows the computed ground state energies versus the number of QDs. It is clear that more QDs in the QDA results in lower ground state energy for a fixed $d_{0}$. Furthermore, for a fixed number of QDs, smaller spacer layer distances lead to lower ground state energies. Right bottom part of Figure 1 shows the differences (in logarithm) 


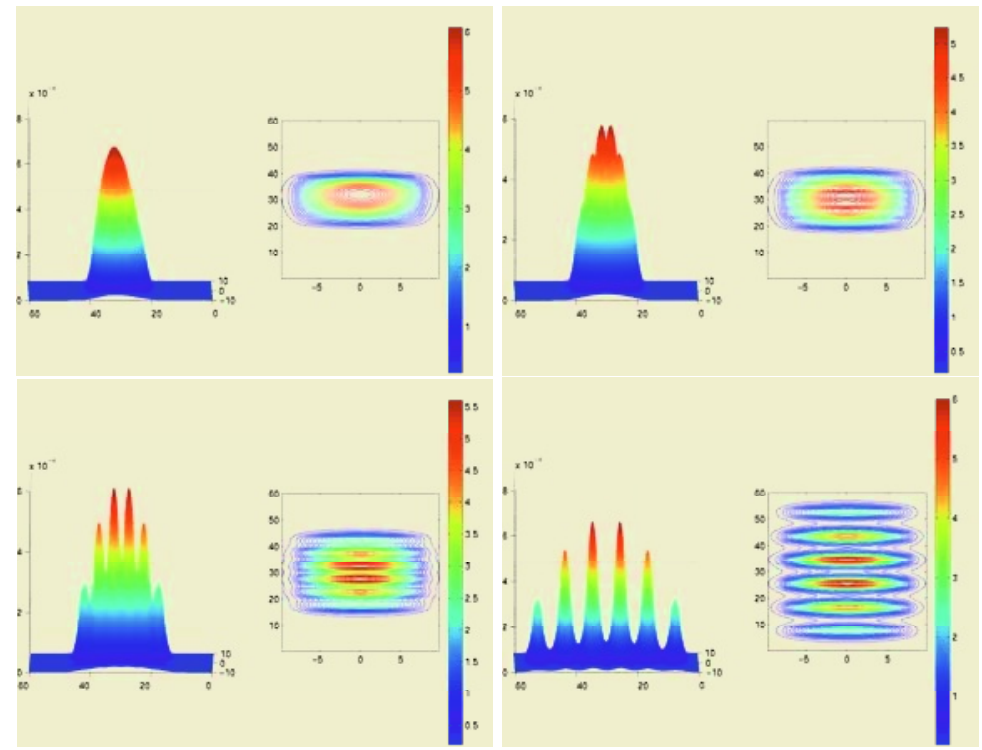

Fig. 2. Wave functions corresponding to the ground state energy. The quantum dot array contains six quantum dots and $d_{0}=0,0.5,2,6 \mathrm{~nm}$, respectively

of the energies for the QDAs containing $n$ and $n+1$ QDs for $n=1, \cdots, 11$. For various $d_{0}=1,2,3 \mathrm{~nm}$, the ground state energies decrease exponentially in a similar manner. To be specific, the energy differences can be nicely fitted by the linearly least-squares lines with slope -0.407 . Figure 2 demonstrates wave functions corresponding to the ground state energy for QDA formed by six quantum dots and $d_{0}=0,0.5,2,6 \mathrm{~nm}$. The results suggest how the wave functions change as the $d_{0}$ is decreased.

\section{References}

1. O. Voskoboynikov, C. P. Lee, and O. Tretyak. Spin-orbit splitting in semiconductor quantum dots with a parabolic confinement potential. Phys. Rev. B, 63:165306, 2001.

2. D. J. BenDaniel and C. B. Duke. Space-charge effects on electron tunnelling. Phys. Rev., 152(683), 1966.

3. M.-C. Lai. A note on finite difference discretizations for Poisson equation on a disk. Numer. Methods Partial Differ. Equ., 17(3):199-203, 2001.

4. W. Wang and T.-M. Hwang and W.-W. Lin and J.-L. Liu. Numerical Methods for Semiconductor Heterostructures with Band Nonparabolicity Journal of Computational Physics, 190(1):141-158, 2003.

5. T.-M. Hwang, W.-W. Lin, J.-L. Liu, and W. Wang. Jacobi-davidson methods for cubic eigenvalue problems. Numerical Linear Algebra with Applications To appear. 